TRANSACTIONS OF THE

AMERICAN MATHEMATICAL SOCIETY

Volume 360, Number 8, August 2008, Pages 4335-4347

S 0002-9947(08)04491-7

Article electronically published on March 14, 2008

\title{
SHARP MORREY-SOBOLEV INEQUALITIES AND THE DISTANCE FROM EXTREMALS
}

\author{
ANDREA CIANCHI
}

\begin{abstract}
Quantitative versions of sharp estimates for the supremum of Sobolev functions in $W^{1, p}\left(\mathbb{R}^{n}\right), p>n$, with remainder terms depending on the distance from the families of extremals, are established.
\end{abstract}

\section{INTRODUCTION AND RESULTS}

The present paper is concerned with the Morrey-Sobolev embedding theorem stating that any weakly differentiable function $u$ in $\mathbb{R}^{n}, n \geq 2$, with $|\nabla u| \in L^{p}\left(\mathbb{R}^{n}\right)$ for some $p>n$, and decaying to 0 at infinity, is essentially bounded (and, in fact, locally Hölder continuous) in $\mathbb{R}^{n}$ (see e.g. [Ad, M2, Z]). A special form of such an embedding tells us that $\|u\|_{L^{\infty}\left(\mathbb{R}^{n}\right)}$ can be estimated in terms of $\|\nabla u\|_{L^{p}\left(\mathbb{R}^{n}\right)}$ under the assumption that

$$
\mathcal{L}^{n}(\operatorname{sprt} u)<+\infty
$$

where $\mathcal{L}^{n}$ denotes the Lebesgue measure in $\mathbb{R}^{n}$, sprt $u$ stands for $\left\{x \in \mathbb{R}^{n}:|u(x)|>\right.$ $0\}$, and $\|\nabla u\|_{L^{p}\left(\mathbb{R}^{n}\right)}$ denotes the $L^{p}\left(\mathbb{R}^{n}\right)$ norm of the length of the gradient $\nabla u$. In particular, an optimal version of the relevant estimate yields

$$
\|u\|_{L^{\infty}\left(\mathbb{R}^{n}\right)} \leq C_{1}(p, n) \mathcal{L}^{n}(\operatorname{sprt} u)^{\frac{1}{n}-\frac{1}{p}}\|\nabla u\|_{L^{p}\left(\mathbb{R}^{n}\right)}
$$

for every $u$ as above fulfilling (1.1) (see e.g. [T2, Theorem 2E]). Here,

$$
C_{1}(p, n)=n^{-1 / p} \omega_{n}^{-1 / n}\left(\frac{p-1}{p-n}\right)^{1 / p^{\prime}},
$$

$\omega_{n}=\pi^{n / 2} / \Gamma(1+n / 2)$, the measure of the unit ball in $\mathbb{R}^{n}$, and $p^{\prime}=\frac{p}{p-1}$, the Hölder conjugate of $p$. The constant $C_{1}(p, n)$ in (1.2) is the best possible, since equality holds whenever $u$ agrees with any of the functions $v_{a, b, x_{0}}: \mathbb{R}^{n} \rightarrow[0,+\infty)$ given by

$$
v_{a, b, x_{0}}(x)= \begin{cases}a\left(b^{\frac{p-n}{p-1}}-\left|x-x_{0}\right|^{\frac{p-n}{p-1}}\right) & \text { if }\left|x-x_{0}\right| \leq b, \\ 0 & \text { otherwise }\end{cases}
$$

for some $a \in \mathbb{R}, b \geq 0$ and $x_{0} \in \mathbb{R}^{n}$.

When assumption (1.1) is dropped, bounds for $\|u\|_{L^{\infty}\left(\mathbb{R}^{n}\right)}$ by $\|\nabla u\|_{L^{p}\left(\mathbb{R}^{n}\right)}$ are possible only in conjunction with some other norm $\|u\|_{L^{q}\left(\mathbb{R}^{n}\right)}$, with $q \in[1, \infty)$. A

Received by the editors August 18, 2006.

2000 Mathematics Subject Classification. Primary 46E35, 46E30.

Key words and phrases. Sobolev inequalities, remainder terms, symmetrizations.

(C)2008 American Mathematical Society Reverts to public domain 28 years from publication 
sharp form of these bounds is available in the endpoint case where $q=1$. Actually, [T2, Theorem $2 \mathrm{C}$ ] tells us that

$$
\|u\|_{L^{\infty}\left(\mathbb{R}^{n}\right)} \leq C_{2}(p, n)\|u\|_{L^{1}\left(\mathbb{R}^{n}\right)}^{1-\eta}\|\nabla u\|_{L^{p}\left(\mathbb{R}^{n}\right)}^{\eta},
$$

for every weakly differentiable function $u \in L^{1}\left(\mathbb{R}^{n}\right)$ such that $|\nabla u| \in L^{p}\left(\mathbb{R}^{n}\right)$. Here,

$$
\eta=\frac{n p}{n p+p-n}
$$

and

$$
C_{2}(p, n)=\left(n \omega_{n}^{1 / n}\right)^{-\frac{n p^{\prime}}{n+p^{\prime}}}\left(\frac{1}{n}+\frac{1}{p^{\prime}}\right)\left(\frac{1}{n}-\frac{1}{p}\right)^{\frac{(n-1) p^{\prime}-n}{n+p^{\prime}}}\left(\frac{\Gamma\left(1+p^{\prime}\right) \Gamma\left(1-p^{\prime} / n^{\prime}\right)}{\Gamma\left(2+p^{\prime} / n\right)}\right)^{n /\left(n+p^{\prime}\right)} .
$$

Furthermore, a family of extremals in (1.5) is given by

$$
w_{a, b, x_{0}}(x)= \begin{cases}a \int_{\left|x-x_{0}\right|}^{b} r^{\frac{1-n}{p-1}}\left(b^{n}-r^{n}\right)^{\frac{1}{p-1}} d r & \text { if }\left|x-x_{0}\right| \leq b, \\ 0 & \text { otherwise }\end{cases}
$$

for $a \in \mathbb{R}, b \geq 0$ and $x_{0} \in \mathbb{R}^{n}$.

Inequalities (1.2) and (1.5) rely upon classical properties of Schwarz symmetrization, and, in particular, on the Pólya-Szegö principle on the decrease of gradient norms under symmetrization. A refined form of this principle, including the characterization of the cases of equality, is contained in [BZ] (see also [FV]), and could be used to show that the $(n+2)$-parameter families of functions given by (1.4) and (1.8) provide, in fact, all the extremals in (1.2) and (1.5), respectively.

Our aim here is to strengthen the above results and to exhibit quantitative versions of (1.2) and (1.5) with a remainder term depending on the distance from extremals. Loosely speaking, we estimate the distance of any function $u$ from the family of extremals $v_{a, b, x_{0}}$ in terms of the gap between the two sides of inequality (1.2), and similarly for inequality (1.5) and its extremals $w_{a, b, x_{0}}$. More precisely, our result concerning (1.2) reads as follows.

Theorem 1.1. Let $p>n$. Then there exist positive constants $\alpha$ and $C_{3}$, depending only on $p$ and $n$, such that

$$
\begin{array}{r}
\|u\|_{L^{\infty}\left(\mathbb{R}^{n}\right)}\left[1+C_{3}\left(\inf _{a, b, x_{0}} \frac{\left\|u-v_{a, b, x_{0}}\right\|_{L^{\infty}\left(\mathbb{R}^{n}\right)}}{\|u\|_{L^{\infty}\left(\mathbb{R}^{n}\right)}}\right)^{\alpha}\right] \\
\leq C_{1}(p, n) \mathcal{L}^{n}(\operatorname{sprt} u)^{\frac{1}{n}-\frac{1}{p}}\|\nabla u\|_{L^{p}\left(\mathbb{R}^{n}\right)}
\end{array}
$$

for every weakly differentiable function $u$ in $\mathbb{R}^{n}$ satisfying (1.1) and such that $|\nabla u| \in$ $L^{p}\left(\mathbb{R}^{n}\right)$. Here, the infimum is extended over all $a \in \mathbb{R}, b \geq 0$ and $x_{0} \in \mathbb{R}^{n}$, and is understood to agree with 0 if $u \equiv 0$.

The counterpart of Theorem 1.1 for inequality (1.5) is contained in the next statement.

Theorem 1.2. Let $p>n$. Then there exist positive constants $\beta$ and $C_{4}$, depending only on $p$ and $n$, such that

$$
\|u\|_{L^{\infty}\left(\mathbb{R}^{n}\right)}\left[1+C_{4}\left(\inf _{a, b, x_{0}} \frac{\left\|u-w_{a, b, x_{0}}\right\|_{L^{\infty}\left(\mathbb{R}^{n}\right)}}{\|u\|_{L^{\infty}\left(\mathbb{R}^{n}\right)}^{\beta}}\right)^{\beta}\right] \leq C_{2}(p, n)\|u\|_{L^{1}\left(\mathbb{R}^{n}\right)}^{1-\eta}\|\nabla u\|_{L^{p}\left(\mathbb{R}^{n}\right)}^{\eta}
$$


for every weakly differentiable function $u \in L^{1}\left(\mathbb{R}^{n}\right)$ such that $|\nabla u| \in L^{p}\left(\mathbb{R}^{n}\right)$. Here, the infimum is defined similarly as in Theorem 1.1.

The remaining part of the paper is devoted to the proofs of Theorems 1.1 and 1.2. which will be accomplished in Sections 2 and 3 , respectively. Let us just recall that Sobolev type inequalities, with optimal constants, are known for $p \in[1, n)$ as well (and are probably even more popular than (1.2) and (1.4)), and go back to $[\mathrm{FF}$ and $\mathrm{M} 1$ in the case when $p=1$, and to $\mathrm{A}$ and $\mathrm{T1}$ in the case when $1<p<n$. Sharp Sobolev and related inequalities involving remainder terms have been the object of a quite rich literature in the last few decades, including BFT, BWW, BL, BN, DHA, FMT, GGS. In particular, our recent paper [C] deals with an inequality estimating the distance from extremals, in the spirit of Theorems 1.1 and 1.2. but in the opposite endpoint situation, having a geometric character, where $p=1$. As far as intermediate values of $p \in(1, n)$ are concerned, a quantitative form of the optimal Sobolev inequality for $p=2$ was established in [BE] by Hilbert spaces and PDE's techniques; the general case requires methods different from those of $[\mathrm{BE},[\mathrm{C}]$ and of this paper, and is the object of [CFMP].

\section{Proof of Theorem 1.1}

Our approach to Theorem 1.1 consists of two steps. First, inequality (1.9) is established for spherically symmetric functions. Second, the (normalized) distance in $L^{\infty}\left(\mathbb{R}^{n}\right)$ of any $u$ from a suitable translate of its Schwarz symmetral $u^{\star}$ is estimated in terms of the gap between the two sides of (1.2). Recall that, given any measurable function $u: \mathbb{R}^{n} \rightarrow \mathbb{R}$ satisfying

$$
\mathcal{L}^{n}(\{|u|>t\})<+\infty \quad \text { for every } t>0,
$$

its decreasing rearrangement $u^{*}:[0,+\infty) \rightarrow[0,+\infty]$ is defined by

$$
u^{*}(s)=\sup \left\{t \geq 0: \mathcal{L}^{n}(\{|u|>t\})>s\right\} \quad \text { for } s \in[0,+\infty),
$$

and its Schwarz symmetral $u^{\star}: \mathbb{R}^{n} \rightarrow[0,+\infty]$ by

$$
u^{\star}(x)=u^{*}\left(\omega_{n}|x|^{n}\right) \quad \text { for } x \in \mathbb{R}^{n} .
$$

The above definitions entail that

$$
\mathcal{L}^{n}\left(\left\{u^{\star}>t\right\}\right)=\mathcal{L}^{1}\left(\left\{u^{*}>t\right\}\right)=\mathcal{L}^{n}(\{|u|>t\}) \quad \text { for } t>0,
$$

whence

$$
\left\|u^{\star}\right\|_{L^{q}\left(\mathbb{R}^{n}\right)}=\left\|u^{*}\right\|_{L^{q}(0, \infty)}=\|u\|_{L^{q}\left(\mathbb{R}^{n}\right)} \quad \text { for every } q \in[1, \infty] .
$$

A much deeper property is provided by the Pólya-Szegö principle, which tells us that if $u$ fulfills (2.1), is weakly differentiable in $\mathbb{R}^{n}$ and $|\nabla u| \in L^{p}\left(\mathbb{R}^{n}\right)$ for some $p \in[1, \infty]$, then $u^{*}$ is locally absolutely continuous in $(0,+\infty), u^{\star}$ is weakly differentiable in $\mathbb{R}^{n}$, and

$$
\left\|\nabla u^{\star}\right\|_{L^{p}\left(\mathbb{R}^{n}\right)} \leq\|\nabla u\|_{L^{p}\left(\mathbb{R}^{n}\right)}
$$

(see e.g. [BZ, [H, [, S, T1]). Moreover, since

$$
\left|\nabla u^{\star}(x)\right|=n \omega_{n}^{1 / n} s^{1 / n^{\prime}}\left(-u^{* \prime}(s)\right) \quad \text { for a.e. } x \in \mathbb{R}^{n},
$$

where $s=\omega_{n}|x|^{n}$, one has

$$
\left\|\nabla u^{\star}\right\|_{L^{p}\left(\mathbb{R}^{n}\right)}=\left\|n \omega_{n}^{1 / n} s^{1 / n^{\prime}}\left(-u^{* \prime}(s)\right)\right\|_{L^{p}(0, \infty)} .
$$


Our discussion of inequality (1.9) for spherically symmetric functions is inspired by the approach of [T2, and requires the following quantitative version of Hölder's inequality.

Lemma 2.1. Let $(X, m)$ be a (positive) measure space, and let $p \geq 2$. Assume that $f \in L^{p}(X, m)$ and $g \in L^{p^{\prime}}(X, m)$, and set

$$
\vartheta=\left(\frac{\int_{X}|g|^{p^{\prime}} d m}{\int_{X}|f|^{p} d m}\right)^{\frac{1}{p p^{\prime}}} .
$$

Then

$$
\int_{X}|f g| d m+\frac{1}{p} \int_{X}\left|\vartheta f-(g / \vartheta)^{\frac{1}{p-1}}\right|^{p} d m \leq\|f\|_{L^{p}}\|g\|_{L^{p^{\prime}}} .
$$

Proof. One has

$$
r s+\frac{1}{p}\left|r-s^{\frac{1}{p-1}}\right|^{p} \leq \frac{r^{p}}{p}+\frac{s^{p^{\prime}}}{p^{\prime}} \quad \text { for every } r, s \geq 0 .
$$

Applying this inequality with $r=\vartheta f$ and $s=g / \vartheta$ and integrating over $X$ yield (2.9).

A key tool in the second step of our proof, dealing with the distance between $u$ and (a suitable translate of) $u^{\star}$, is a quantitative form of inequality (2.5), recently proved in [CEFT, Theorem 4.1] (see also CF for the one-dimensional case), which reads as follows. Let $n \geq 1$ and let $p>1$. Given any weakly differentiable function $u$ satisfying (1.1) and such that $|\nabla u| \in L^{p}\left(\mathbb{R}^{n}\right)$, define

$$
\begin{aligned}
& E(u)=\frac{\int_{\mathbb{R}^{n}}|\nabla u|^{p} d x}{\int_{\mathbb{R}^{n}}\left|\nabla u^{\star}\right| p d x}-1, \\
& \quad f\left|\nabla u^{\star}\right|^{p} d x=\frac{1}{\mathcal{L}^{n}(\operatorname{sprt} u)} \int_{\mathbb{R}^{n}}\left|\nabla u^{\star}\right|^{p} d x
\end{aligned}
$$

and

$$
M_{u^{\star}}(\sigma)=\frac{\mathcal{L}^{n}\left(\left\{\left|\nabla u^{\star}\right| \leq \sigma\right\} \cap\left\{0<u^{\star}<\operatorname{esssup}|u|\right\}\right)}{\mathcal{L}^{n}(\operatorname{sprt} u)} \quad \text { for } \sigma>0 .
$$

Then there exist positive constants $r_{1}, r_{2}, r_{3}$ and $C$, depending only on $p$ and $n$, such that

$$
\begin{aligned}
& \text { (2.10) } \min _{ \pm} \inf _{x_{0} \in \mathbb{R}^{n}}\left\|u(\cdot) \pm u^{\star}\left(\cdot-x_{0}\right)\right\|_{L^{1}\left(\mathbb{R}^{n}\right)} \\
& \leq C\left(f\left|\nabla u^{\star}\right|^{p} d x\right)^{1 / p} \mathcal{L}^{n}(\operatorname{sprt} u)^{1+\frac{1}{n}}\left[M_{u^{\star}}(\sigma)+E(u)^{r_{1}}+\frac{\left(\left.f\left|\nabla u^{\star}\right|\right|^{p} d x\right)^{1 / p}}{\sigma} E(u)^{r_{2}}\right]^{r_{3}}
\end{aligned}
$$

for every $\sigma>0$. Notice that, in particular, the exponents $r_{1}, r_{2}$ and $r_{3}$ in (2.10) can be chosen arbitrarily small.

Proof of Theorem 1.1. Assume, for the time being, that

$$
\mathcal{L}^{n}(\operatorname{sprt} u)=1
$$

and

$$
\|u\|_{L^{\infty}\left(\mathbb{R}^{n}\right)}=1
$$


Set

$$
\epsilon=C_{1}(p, n)^{p}\|\nabla u\|_{L^{p}\left(\mathbb{R}^{n}\right)}^{p}-1 .
$$

Owing to (2.5), (2.4) and (1.2),

$$
0 \leq\|\nabla u\|_{L^{p}\left(\mathbb{R}^{n}\right)}^{p}-\left\|\nabla u^{\star}\right\|_{L^{p}\left(\mathbb{R}^{n}\right)}^{p} \leq \frac{\epsilon}{C_{1}(p, n)^{p}}
$$

and

$$
0 \leq C_{1}(p, n)\left\|\nabla u^{\star}\right\|_{L^{p}\left(\mathbb{R}^{n}\right)}-1 \leq C_{1}(p, n)^{p}\left\|\nabla u^{\star}\right\|_{L^{p}\left(\mathbb{R}^{n}\right)}^{p}-1 \leq \epsilon .
$$

Inequality (2.14) will be used to estimate $\min _{ \pm} \inf _{x_{0}}\left\|u(\cdot) \pm u^{\star}\left(\cdot-x_{0}\right)\right\|_{L^{\infty}\left(\mathbb{R}^{n}\right)}$, whereas (2.15) will provide an estimate for $\inf _{a, b}\left\|u^{\star}\left(\cdot-x_{0}\right)-v_{a, b, x_{0}}(\cdot)\right\|_{L^{\infty}\left(\mathbb{R}^{n}\right)}$. These estimates will immediately lead to (1.9). Consider the latter first. Define

$$
\lambda=C_{1}(p, n)^{\frac{1}{p}}\left(\int_{0}^{1}\left(n \omega_{n}^{1 / n} s^{1 / n^{\prime}}\left(-u^{* \prime}(s)\right)\right)^{p} d s\right)^{-\frac{1}{p p^{\prime}}}
$$

and

$$
\Lambda=n \omega_{n}^{1 / n} \lambda .
$$

Equations (2.4) and (2.12), an application of Lemma 2.1, and (2.7) tell us that

$$
\begin{aligned}
& 1=u^{*}(0)= \int_{0}^{1}\left(-u^{* \prime}(s)\right) d s \\
& \leq \frac{1}{n \omega_{n}^{1 / n}}\left(\int_{0}^{1}\left(n \omega_{n}^{1 / n} s^{1 / n^{\prime}}\left(-u^{* \prime}(s)\right)\right)^{p} d s\right)^{1 / p}\left(\int_{0}^{1} s^{-p^{\prime} / n^{\prime}} d s\right)^{1 / p^{\prime}} \\
& \quad \quad-\frac{1}{p} \int_{0}^{1}\left|\Lambda s^{1 / n^{\prime}}\left(-u^{* \prime}(s)\right)-\left(s^{-1 / n^{\prime}} / \Lambda\right)^{\frac{1}{p-1}}\right|^{p} d s \\
&=C_{1}(p, n)\left\|\nabla u^{\star}\right\|_{L^{p}\left(\mathbb{R}^{n}\right)}-\frac{1}{p} \int_{0}^{1}\left|\Lambda s^{1 / n^{\prime}}\left(-u^{* \prime}(s)\right)-\left(s^{-1 / n^{\prime}} / \Lambda\right)^{\frac{1}{p-1}}\right|^{p} d s .
\end{aligned}
$$

Coupling (2.15) and (2.18) yields

$$
\int_{0}^{1}\left|\Lambda s^{1 / n^{\prime}}\left(-u^{* \prime}(s)\right)-\left(s^{-1 / n^{\prime}} / \Lambda\right)^{\frac{1}{p-1}}\right|^{p} d s \leq p \epsilon .
$$

Now, define $\phi:[0,1] \rightarrow[0, \infty)$ as

$$
\phi(s)=\frac{1-s^{1-p^{\prime} / n^{\prime}}}{\Lambda^{p^{\prime}}\left(1-p^{\prime} / n^{\prime}\right)} \quad \text { for } s \in[0,1] .
$$

Then,

$$
\phi\left(\omega_{n}|x|^{n}\right)=v_{a, b, 0}(x) \quad \text { if } \omega_{n}|x|^{n} \leq 1,
$$

provided that $a$ and $b$ are suitably chosen. Since $u^{*}(1)=\phi(1)=0$,

$$
\begin{aligned}
\left\|u^{*}-\phi\right\|_{L^{\infty}(0,1)} & \leq \int_{0}^{1}\left|u^{* \prime}(s)-\phi^{\prime}(s)\right| d s \\
& \leq\left(\int_{0}^{1}\left|s^{1 / n^{\prime}}\left(-u^{* \prime}(s)\right)-s^{-1 /\left(n^{\prime}(p-1)\right)} \Lambda^{-p^{\prime}}\right|^{p} d s\right)^{\frac{1}{p}}\left(\int_{0}^{1} s^{-p^{\prime} / n^{\prime}} d s\right)^{\frac{1}{p^{\prime}}} .
\end{aligned}
$$


Hence, by (2.19) and (2.17),

$$
\left\|u^{*}-\phi\right\|_{L^{\infty}(0,1)} \leq C_{5} \epsilon^{\frac{1}{p}}\left(\int_{0}^{1}\left(n \omega_{n}^{1 / n} s^{1 / n^{\prime}}\left(-u^{* \prime}(s)\right)\right)^{p} d s\right)^{\frac{1}{p p^{\prime}}}
$$

for some constant $C_{5}$ depending only on $p$ and $n$. Owing to (2.7) and (2.15), inequality (2.21) entails that, for every $x_{0} \in \mathbb{R}^{n}$,

$$
\inf _{a, b}\left\|u^{\star}\left(\cdot-x_{0}\right)-v_{a, b, x_{0}}(\cdot)\right\|_{L^{\infty}\left(\mathbb{R}^{n}\right)} \leq C_{5} \epsilon^{\frac{1}{p}}\left((1+\epsilon) / C_{1}(p, n)\right)^{\frac{1}{p^{\prime}}} .
$$

When $\epsilon \leq 1$, inequality (2.22) yields

$$
\inf _{a, b}\left\|u^{\star}\left(\cdot-x_{0}\right)-v_{a, b, x_{0}}(\cdot)\right\|_{L^{\infty}\left(\mathbb{R}^{n}\right)} \leq C_{5} \epsilon^{\frac{1}{p}}\left(2 / C_{1}(p, n)\right)^{\frac{1}{p^{\prime}}}
$$

for every $x_{0} \in \mathbb{R}^{n}$. On the other hand, if $\epsilon>1$, then trivially

$$
\inf _{a, b}\left\|u^{\star}\left(\cdot-x_{0}\right)-v_{a, b, x_{0}}(\cdot)\right\|_{L^{\infty}\left(\mathbb{R}^{n}\right)} \leq\left\|u^{\star}\right\|_{L^{\infty}\left(\mathbb{R}^{n}\right)}=1 \leq \epsilon^{\frac{1}{p}}
$$

for every $x_{0} \in \mathbb{R}^{n}$. Thus, there exists a constant $C_{6}$, depending only on $p$ and $n$, such that, under (2.11) and (2.12),

$$
\inf _{a, b}\left\|u^{\star}\left(\cdot-x_{0}\right)-v_{a, b, x_{0}}(\cdot)\right\|_{L^{\infty}\left(\mathbb{R}^{n}\right)} \leq C_{6} \epsilon^{\frac{1}{p}}
$$

for every $x_{0} \in \mathbb{R}^{n}$.

Now we estimate $\min _{ \pm} \inf _{x_{0}}\left\|u(\cdot) \pm u^{\star}\left(\cdot-x_{0}\right)\right\|_{L^{\infty}\left(\mathbb{R}^{n}\right)}$. It is at this stage that the quantitative Pólya-Szegö inequality (2.10) comes into play. Let $r_{1}, r_{2}, r_{3}$ be any positive numbers which make (2.10) true. By (2.6),

$$
M_{u^{\star}}(\sigma) \leq \mathcal{L}^{1}\left(\left\{s \in[0,1]: n \omega_{n}^{1 / n} s^{1 / n^{\prime}}\left(-u^{* \prime}(s)\right) \leq \sigma\right\}\right) \quad \text { for every } \sigma>0 .
$$

Thanks to inequality (2.24) and to the inclusion

$$
\{\varphi \leq t\} \subset\{|\varphi-\psi| \geq t\} \cup\{\psi \leq 2 t\},
$$

which holds for any functions $\varphi, \psi:[0,1] \rightarrow[0,+\infty)$ and every $t>0$, we get that

$$
\begin{aligned}
M_{u \star}(\sigma) \leq \mathcal{L}^{1}\left(\left\{s \in[0,1]:\left|\Lambda s^{1 / n^{\prime}}\left(-u^{* \prime}(s)\right)-\left(s^{-1 / n^{\prime}} / \Lambda\right)^{\frac{1}{p-1}}\right| \geq \lambda \sigma\right\}\right) \\
+\quad \mathcal{L}^{1}\left(\left\{s \in[0,1]:\left(s^{-1 / n^{\prime}} / \Lambda\right)^{\frac{1}{p-1}} \leq 2 \lambda \sigma\right\}\right) \text { for every } \sigma>0 .
\end{aligned}
$$

By (2.19),

$$
\begin{aligned}
& \mathcal{L}^{1}\left(\left\{s \in[0,1]:\left|\Lambda s^{1 / n^{\prime}}\left(-u^{* \prime}(s)\right)-\left(s^{-1 / n^{\prime}} / \Lambda\right)^{\frac{1}{p-1}}\right|\right.\right. \\
& \quad \geq \lambda \sigma\}) \leq \frac{p \epsilon}{(\lambda \sigma)^{p}} \quad \text { for every } \sigma>0 .
\end{aligned}
$$

On the other hand,

$$
\begin{aligned}
\mathcal{L}^{1}(\{s & \left.\left.\in[0,1]:\left(s^{-1 / n^{\prime}} / \Lambda\right)^{\frac{1}{p-1}} \leq 2 \lambda \sigma\right\}\right) \\
& \leq \max \left\{1-\left[(2 \lambda \sigma)^{p-1} \Lambda\right]^{-n^{\prime}}, 0\right\} \leq\left[(2 \lambda \sigma)^{p-1} \Lambda\right]^{n^{\prime}} \quad \text { for every } \sigma>0 .
\end{aligned}
$$


Combining (2.25)-(2.27) yields

$$
M_{u} \star(\sigma) \leq \frac{p \epsilon}{(\lambda \sigma)^{p}}+\left[(2 \lambda \sigma)^{p-1} \Lambda\right]^{n^{\prime}} \quad \text { for every } \sigma>0 .
$$

Let $\gamma$ be a number in $(0,1)$ so close to 1 that $r_{2}-\frac{1-\gamma}{p}>0$, where $r_{2}$ is the exponent appearing in (2.10). On choosing $\sigma=\frac{1}{\lambda} \epsilon^{\frac{1-\gamma}{p}}$ in (2.28), and observing that

$$
\lambda=C_{1}(p, n)^{1 / p}\left\|\nabla u^{\star}\right\|_{L^{p}\left(\mathbb{R}^{n}\right)}^{-1 / p^{\prime}} \leq C_{1}(p, n)\left\|u^{\star}\right\|_{L^{\infty}\left(\mathbb{R}^{n}\right)}^{-1 / p^{\prime}}=C_{1}(p, n),
$$

we obtain

$$
M_{u} \star\left(\epsilon^{\frac{1-\gamma}{p}} / \lambda\right) \leq p \epsilon^{\gamma}+\left(2^{p-1} n \omega_{n}^{1 / n} C_{1}(p, n) \epsilon^{\frac{1-\gamma}{p^{\prime}}}\right)^{n^{\prime}} .
$$

By (2.14) and (2.15),

$$
E(u) \leq \frac{\epsilon}{C_{1}(p, n)^{p}\left\|\nabla u^{\star}\right\|_{L^{p}\left(\mathbb{R}^{n}\right)}^{p}} \leq \epsilon .
$$

Hence, owing to (2.5) and (2.31),

$$
\left(f\left|\nabla u^{\star}\right|^{p} d x\right)^{1 / p} \epsilon^{\frac{\gamma-1}{p}} E(u)^{r_{2}} \leq\|\nabla u\|_{L^{p}\left(\mathbb{R}^{n}\right)} \epsilon^{r_{2}-\frac{1-\gamma}{p}} .
$$

From (2.10) applied with $\sigma=\frac{1}{\lambda} \epsilon^{\frac{1-\gamma}{p}}$, one deduces via (2.5), (2.30), (2.31), (2.32) and (2.29) that a constant $C_{7}$, depending only on $p$ and $n$, exists such that

$$
\begin{aligned}
& \min _{ \pm} \inf _{x_{0}}\left\|u(\cdot) \pm u^{\star}\left(\cdot-x_{0}\right)\right\|_{L^{1}\left(\mathbb{R}^{n}\right)} \\
& \quad \leq C_{7}\|\nabla u\|_{L^{p}\left(\mathbb{R}^{n}\right)}\left(\epsilon^{\gamma}+\epsilon^{\frac{1-\gamma}{p^{\prime}} n^{\prime}}+\epsilon^{r_{1}}+\|\nabla u\|_{L^{p}\left(\mathbb{R}^{n}\right)} \epsilon^{r_{2}-\frac{1-\gamma}{p}}\right)^{r_{3}} .
\end{aligned}
$$

To fix ideas, let us suppose that $\min _{ \pm}$is attained in (2.33) with the minus sign, the other case being completely analogous. Assume, for a moment, that $\epsilon \leq 1$. Then, by (2.33) and (2.13), positive constants $C_{8}$ and $\delta$, depending only on $p$ and $n$, exist such that

$$
\inf _{x_{0}}\left\|u(\cdot)-u^{\star}\left(\cdot-x_{0}\right)\right\|_{L^{1}\left(\mathbb{R}^{n}\right)} \leq C_{8} \epsilon^{\delta} .
$$

Inequalities (1.5) and (2.5) entail that, for every $x_{0} \in \mathbb{R}^{n}$,

$$
\begin{aligned}
\| u(\cdot) & -u^{\star}\left(\cdot-x_{0}\right) \|_{L^{\infty}\left(\mathbb{R}^{n}\right)} \\
& \leq C_{2}(p, n)\left\|u(\cdot)-u^{\star}\left(\cdot-x_{0}\right)\right\|_{L^{1}\left(\mathbb{R}^{n}\right)}^{1-\eta}\left\|\nabla\left[u(\cdot)-u^{\star}\left(\cdot-x_{0}\right)\right]\right\|_{L^{p}\left(\mathbb{R}^{n}\right)}^{\eta} \\
& \leq 2^{\eta} C_{2}(p, n)\left\|u(\cdot)-u^{\star}\left(\cdot-x_{0}\right)\right\|_{L^{1}\left(\mathbb{R}^{n}\right)}^{1-\eta}\|\nabla u\|_{L^{p}\left(\mathbb{R}^{n}\right)}^{\eta} .
\end{aligned}
$$

On exploiting again the fact that $\epsilon \leq 1$, we deduce from (2.13), (2.34) and (2.35) that

$$
\inf _{x_{0}}\left\|u(\cdot)-u^{\star}\left(\cdot-x_{0}\right)\right\|_{L^{\infty}\left(\mathbb{R}^{n}\right)} \leq C_{9} \epsilon^{\delta(1-\eta)}
$$


for some positive constant $C_{9}$ depending only on $p$ and $n$. Combining (2.36) and (2.23) tells us that

$$
\begin{aligned}
\inf _{a, b, x_{0}} & \left\|u-v_{a, b, x_{0}}\right\|_{L^{\infty}\left(\mathbb{R}^{n}\right)} \\
\leq & \inf _{x_{0}}\left(\left\|u(\cdot)-u^{\star}\left(\cdot-x_{0}\right)\right\|_{L^{\infty}\left(\mathbb{R}^{n}\right)}+\inf _{a, b}\left\|u^{\star}\left(\cdot-x_{0}\right)-v_{a, b, x_{0}}(\cdot)\right\|_{L^{\infty}\left(\mathbb{R}^{n}\right)}\right) \\
\leq & C_{9} \epsilon^{\delta(1-\eta)}+C_{6} \epsilon^{1 / p} \leq\left(C_{9}+C_{6}\right) \epsilon^{\nu},
\end{aligned}
$$

if $u$ satisfies (2.11), (2.12) and $\epsilon \leq 1$. Here $\nu=\min \{1 / p, \delta(1-\eta)\}$. Since a constant $C$ exists such that $s^{p}-1 \leq C(s-1)$ if $0 \leq s \leq 2^{1 / p}$, inequality (2.37) ensures that

$$
\inf _{a, b, x_{0}}\left\|u-v_{a, b, x_{0}}\right\|_{L^{\infty}\left(\mathbb{R}^{n}\right)} \leq C_{10}\left(C_{1}(p, n)\|\nabla u\|_{L^{p}\left(\mathbb{R}^{n}\right)}-1\right)^{\nu}
$$

for some positive constant $C_{10}$ depending only on $p$ and $n$, and for every function $u$ as in the statement satisfying, in addition, (2.11), (2.12) and $\epsilon \leq 1$. An inequality of type (2.38) trivially continues to hold even if $\epsilon>1$, provided that (2.11) and (2.12) are in force, since in this case

$$
\begin{aligned}
\inf _{a, b, x_{0}}\left\|u-v_{a, b, x_{0}}\right\|_{L^{\infty}\left(\mathbb{R}^{n}\right)}^{1 / \nu} & \leq \inf _{a, b, x_{0}}\left\|u-v_{a, b, x_{0}}\right\|_{L^{\infty}\left(\mathbb{R}^{n}\right)} \\
& \leq 1 \leq \frac{1}{2^{1 / p}-1}\left(C_{1}(p, n)\|\nabla u\|_{L^{p}\left(\mathbb{R}^{n}\right)}-1\right) .
\end{aligned}
$$

Finally, if $u$ is just as in the statement, then an application of (2.38) to the function $\bar{u}$ given by

$$
\bar{u}(x)=\frac{u(c x)}{\|u\|_{L^{\infty}\left(\mathbb{R}^{n}\right)}} \quad \text { for } x \in \mathbb{R}^{n},
$$

where $c=\mathcal{L}^{n}(\operatorname{sprt} u)^{1 / n}$ (a function fulfilling (2.11) and (2.12) $)$, yields (1.9), with $\alpha=1 / \nu$.

\section{Proof of Theorem 1.2}

The outline of the proof of Theorem 1.2 is similar to that of Theorem 1.1. However, some complications arise, owing to the fact that Theorem 1.2 deals with a multiplicative inequality, and that functions whose support need not have finite measure are involved.

Proof of Theorem 1.2, Set

$$
C_{11}=\frac{1}{n \omega_{n}^{1 / n}}\left(\frac{\Gamma\left(1+p^{\prime}\right) \Gamma\left(1-p^{\prime} / n^{\prime}\right)}{\Gamma\left(2+p^{\prime} / n\right)}\right)^{1 / p^{\prime}} .
$$

Assume, for the time being, that $u$ satisfies the additional conditions (2.12) and

$$
\|u\|_{L^{1}\left(\mathbb{R}^{n}\right)}=C_{11}\left(\frac{1}{n}-\frac{1}{p}\right)\left\|\nabla u^{\star}\right\|_{L^{p}\left(\mathbb{R}^{n}\right)} .
$$

Define

$$
\delta=C_{2}(p, n)^{p / \eta}\|u\|_{L^{1}\left(\mathbb{R}^{n}\right)}^{\frac{(1-\eta) p}{\eta}}\left\|\nabla u^{\star}\right\|_{L^{p}\left(\mathbb{R}^{n}\right)}^{p}-1 .
$$

Then, by (2.5), (2.4) and (1.5),

$$
0 \leq C_{2}(p, n)^{p / \eta}\|u\|_{L^{1}\left(\mathbb{R}^{n}\right)}^{\frac{(1-\eta) p}{\eta}}\left(\|\nabla u\|_{L^{p}\left(\mathbb{R}^{n}\right)}^{p}-\left\|\nabla u^{\star}\right\|_{L^{p}\left(\mathbb{R}^{n}\right)}^{p}\right) \leq \delta
$$


and

$$
\begin{aligned}
0 & \leq C_{2}(p, n)\left\|u^{\star}\right\|_{L^{1}\left(\mathbb{R}^{n}\right)}^{1-\eta}\left\|\nabla u^{\star}\right\|_{L^{p}\left(\mathbb{R}^{n}\right)}^{\eta}-1 \\
& \leq C_{2}(p, n)^{p / \eta}\left\|u^{\star}\right\|_{L^{1}\left(\mathbb{R}^{n}\right)}^{\frac{(1-\eta) p}{\eta}}\left\|\nabla u^{\star}\right\|_{L^{p}\left(\mathbb{R}^{n}\right)}^{p}-1 \leq \delta .
\end{aligned}
$$

Next set

$$
\theta=\frac{1}{\left(n \omega_{n}^{1 / n}\right)^{1 / p}}\left(\int_{0}^{1}\left((1-s) s^{-1 / n^{\prime}}\right)^{p^{\prime}} d s\right)^{\frac{1}{p p^{\prime}}}\left(\int_{0}^{1}\left(n \omega_{n}^{1 / n} s^{1 / n^{\prime}}\left(-u^{* \prime}(s)\right)\right)^{p} d s\right)^{-\frac{1}{p p^{\prime}}}
$$

and observe that

$$
\theta=C_{11}^{1 / p}\left(\int_{\left\{u^{\star}>u^{*}(1)\right\}}\left|\nabla u^{\star}\right|^{p} d x\right)^{-\frac{1}{p p^{\prime}}} ;
$$

moreover, define

$$
\Theta=n \omega_{n}^{1 / n} \theta
$$

The following chain holds:

$$
\begin{aligned}
& 1= u^{*}(0)=\int_{0}^{1} u^{*}(s) d s+\int_{0}^{1}(1-s)\left(-u^{* \prime}(s)\right) d s \\
& \leq \int_{0}^{1} u^{*}(s) d s+\frac{1}{n \omega_{n}^{1 / n}}\left(\int_{0}^{1}\left((1-s) s^{-1 / n^{\prime}}\right)^{p^{\prime}} d s\right)^{\frac{1}{p^{\prime}}}\left(\int_{0}^{1}\left(n \omega_{n}^{1 / n} s^{1 / n^{\prime}}\left(-u^{* \prime}(s)\right)\right)^{p} d s\right)^{\frac{1}{p}} \\
& \quad-\frac{1}{p} \int_{0}^{1}\left|\Theta s^{1 / n^{\prime}}\left(-u^{*^{\prime}}(s)\right)-\left((1-s) s^{-1 / n^{\prime}} / \Theta\right)^{\frac{1}{p-1}}\right|^{p} d s \\
&= \int_{0}^{\infty} u^{*}(s) d s+C_{11}\left(\int_{0}^{\infty}\left(n \omega_{n}^{1 / n} s^{1 / n^{\prime}}\left(-u^{* \prime}(s)\right)\right)^{p} d s\right)^{\frac{1}{p}}-\int_{1}^{\infty} u^{*}(s) d s \\
&-C_{11}\left[\left(\int_{0}^{\infty}\left(n \omega_{n}^{1 / n} s^{1 / n^{\prime}}\left(-u^{* \prime}(s)\right)\right)^{p} d s\right)^{\frac{1}{p}}-\left(\int_{0}^{1}\left(n \omega_{n}^{1 / n} s^{1 / n^{\prime}}\left(-u^{* \prime}(s)\right)\right)^{p} d s\right)^{\frac{1}{p}}\right] \\
&= C_{2}(p, n)\left\|u^{\star}\right\|_{L^{1}\left(\mathbb{R}^{n}\right)}^{1-\eta} \mid \nabla u^{\star} \|_{L^{p}\left(\mathbb{R}^{n}\right)}^{\eta}-\int_{1}^{\infty} u^{*}(s) d s \\
&-C_{11}\left[\left(\int_{0}^{\infty}\left(n \omega_{n}^{1 / n} s^{1 / n^{\prime}}\left(-u^{* \prime}(s)\right)\right)^{p} d s\right)^{\frac{1}{p}}-\left(\int_{0}^{1}\left(n \omega_{n}^{1 / n} s^{1 / n^{\prime}}\left(-u^{* \prime}(s)\right)\right)^{p} d s\right)^{\frac{1}{p}}\right] \\
& \quad-\frac{1}{p} \int_{0}^{1}\left|\Theta s^{1 / n^{\prime}}\left(-u^{* \prime}(s)\right)-\left((1-s) s^{-1 / n^{\prime}} / \Theta\right)^{\frac{1}{p-1}}\right|^{p} d s,
\end{aligned}
$$

where the inequality is a consequence of Lemma 2.1] and the last equality of (3.1).

From (3.4) and (3.7) we deduce that

$$
\begin{gathered}
\int_{0}^{1}\left|\Theta s^{1 / n^{\prime}}\left(-u^{* \prime}(s)\right)-\left((1-s) s^{-1 / n^{\prime}} / \Theta\right)^{\frac{1}{p-1}}\right|^{p} d s \leq p \delta \\
\int_{1}^{\infty} u^{*}(s) d s \leq \delta
\end{gathered}
$$


and

$$
\int_{1}^{\infty}\left(n \omega_{n}^{1 / n} s^{1 / n^{\prime}}\left(-u^{* \prime}(s)\right)\right)^{p} d s \leq \frac{p \delta}{C_{11}}\left(\int_{0}^{\infty}\left(n \omega_{n}^{1 / n} s^{1 / n^{\prime}}\left(-u^{* \prime}(s)\right)\right)^{p} d s\right)^{\frac{1}{p}}
$$

Notice that, in deriving (3.10), we have made use of the fact that $p\left(s^{1 / p}-r^{1 / p}\right) \geq$ $(s-r) s^{-1 / p^{\prime}}$ if $0<r \leq s$.

Define $\psi:[0, \infty] \rightarrow[0, \infty)$ as

$$
\psi(s)= \begin{cases}\int_{s}^{1}(1-r)^{\frac{1}{p-1}} r^{-\frac{p^{\prime}}{n^{\prime}}} \Theta^{-p^{\prime}} d r & \text { if } s \in[0,1], \\ 0 & \text { otherwise }\end{cases}
$$

Hence,

$$
\psi\left(\omega_{n}|x|^{n}\right)=w_{a, b, 0}(x) \quad \text { for } x \in \mathbb{R}^{n},
$$

and for an appropriate choice of $a$ and $b$. Then, via an analogous argument as in the proof of (2.21), inequality (3.8) entails that

$$
\left\|u^{*}-u^{*}(1)-\psi\right\|_{L^{\infty}(0,1)} \leq C_{12} \delta^{\frac{1}{p}}\left(\int_{0}^{\infty}\left(n \omega_{n}^{1 / n} s^{1 / n^{\prime}}\left(-u^{* \prime}(s)\right)\right)^{p} d s\right)^{\frac{1}{p p^{\prime}}}
$$

for some positive constant $C_{12}$ depending only on $p$ and $n$. Hence, by (3.1) and (3.4), one gets

$$
\left\|u^{*}-u^{*}(1)-\psi\right\|_{L^{\infty}(0,1)} \leq C_{13} \delta^{1 / p}(1+\delta)^{1 / p^{\prime}}
$$

for some positive constant $C_{13}$ depending only on $p$ and $n$. On the other hand, defining $U:[0, \infty) \rightarrow[0, \infty)$ as

$$
U(s)=u^{*}(s+1) \quad \text { for } s \geq 0,
$$

one has by (3.7) applied with $u^{*}$ replaced by $U$

$$
U(0) \leq C_{2}(p, n)\left(\int_{0}^{\infty} U(s) d s\right)^{1-\eta}\left(\int_{0}^{\infty}\left(n \omega_{n}^{1 / n} s^{1 / n^{\prime}}\left(-U^{\prime}(s)\right)\right)^{p} d s\right)^{\frac{\eta}{p}} .
$$

Thus,

$$
\begin{aligned}
u^{*}(1) & \leq C_{2}(p, n)\left(\int_{1}^{\infty} u^{*}(s) d s\right)^{1-\eta}\left(\int_{1}^{\infty}\left(n \omega_{n}^{1 / n} s^{1 / n^{\prime}}\left(-u^{* \prime}(s)\right)\right)^{p} d s\right)^{\frac{\eta}{p}} \\
& \leq C_{2}(p, n) \delta^{1-\eta}\left(\frac{p \delta}{C_{11}}\left\|\nabla u^{\star}\right\|_{L^{p}\left(\mathbb{R}^{n}\right)}^{p-1}\right)^{\frac{\eta}{p}} \\
& =C_{14} \delta^{1-\eta / p^{\prime}}\left(\left\|u^{\star}\right\|_{L^{1}\left(\mathbb{R}^{n}\right)}^{1-\eta}\left\|\nabla u^{\star}\right\|_{L^{p}\left(\mathbb{R}^{n}\right)}^{\eta}\right)^{\frac{\eta}{p^{\prime}}} \\
& \leq C_{14} \delta^{1-\eta / p^{\prime}}\left((1+\delta) / C_{2}(p, n)\right)^{\frac{\eta}{p^{\prime}}}
\end{aligned}
$$

for some positive constant $C_{14}$ depending only on $p$ and $n$. Notice that the second inequality is a consequence of (3.9) and (3.10), the equality of (3.1), and the last inequality of (3.4). Inequalities (3.11) and (3.13), and the fact that $1-\eta / p^{\prime}>1 / p$, easily imply that a constant $C_{15}$, depending only on $p$ and $n$, exists such that

$$
\inf _{a, b}\left\|u^{\star}\left(\cdot-x_{0}\right)-w_{a, b, x_{0}}(\cdot)\right\|_{L^{\infty}\left(\mathbb{R}^{n}\right)} \leq C_{15} \delta^{\frac{1}{p}}
$$

for every $x_{0} \in \mathbb{R}^{n}$.

Our next task is to estimate $\min _{ \pm} \inf _{x_{0}}\left\|u(\cdot) \pm u^{\star}\left(\cdot-x_{0}\right)\right\|_{L^{\infty}\left(\mathbb{R}^{n}\right)}$. Define $u_{1}$ and $u_{2}: \mathbb{R}^{n} \rightarrow \mathbb{R}$ as

$$
u_{1}(x)=\operatorname{sign}(u(x)) \max \left\{|u(x)|-u^{*}(1), 0\right\}
$$


and

$$
u_{2}(x)=\operatorname{sign}(u(x)) \min \left\{|u(x)|, u^{*}(1)\right\}
$$

for $x \in \mathbb{R}^{n}$, so that $u=u_{1}+u_{2}$. A similar argument as in the proof of (2.28), exploiting (3.8), enables us to derive that

$$
M_{u_{1} \star}(\sigma) \leq \frac{p \delta}{(\theta \sigma)^{p}}+C_{16}\left[\left((\theta \sigma)^{p-1} \Theta\right)^{n^{\prime}}+(\theta \sigma)^{p-1} \Theta\right] \quad \text { for every } \sigma>0,
$$

for some absolute constant $C_{16}$. We skip the details of this derivation. Let us limit ourselves to mentioning that here we have made use of the inclusion

$$
\begin{aligned}
& \left\{s \in[0,1]:(1-s)^{\frac{1}{p-1}} s^{-\frac{1}{n^{\prime}(p-1)}} \Theta^{-\frac{1}{p-1}} \leq t\right\} \\
& \subset\left\{s \in[0,1 / 2]: s^{-\frac{1}{n^{\prime}(p-1)}}(2 \Theta)^{-\frac{1}{p-1}} \leq t\right\} \cup\left\{s \in[1 / 2,1]:(1-s)^{\frac{1}{p-1}} \Theta^{-\frac{1}{p-1}} \leq t\right\}
\end{aligned}
$$

for $t>0$. Inequality (3.15), with $\sigma=\frac{1}{\theta} \delta^{\frac{1-\gamma}{p}}$ and $\gamma \in(0,1)$ to be fixed later, and equation (3.5) entail that

$$
\begin{aligned}
& M_{u_{1} \star}\left(\delta^{\frac{1-\gamma}{p}} / \theta\right) \\
& \leq p \delta^{\gamma}+C_{17}\left[\delta^{\frac{(1-\gamma) n^{\prime}}{p^{\prime}}}\left(\int_{\left\{u^{\star}>u^{*}(1)\right\}}\left|\nabla u^{\star}\right|^{p} d x\right)^{-\frac{n^{\prime}}{p p^{\prime}}}\right. \\
& \left.\quad+\delta^{\frac{1-\gamma}{p^{\prime}}}\left(\int_{\left\{u^{\star}>u^{*}(1)\right\}}\left|\nabla u^{\star}\right|^{p} d x\right)^{-\frac{1}{p p^{\prime}}}\right]
\end{aligned}
$$

for some constant $C_{17}$ depending only on $p$ and $n$. On the other hand,

$$
\begin{aligned}
E\left(u_{1}\right) & =\frac{\int_{\left\{|u|>u^{*}(1)\right\}}|\nabla u|^{p} d x-\int_{\left\{u^{\star}>u^{*}(1)\right\}}\left|\nabla u^{\star}\right|^{p} d x}{\int_{\left\{u^{\star}>u^{*}(1)\right\}}\left|\nabla u^{\star}\right|^{p} d x} \\
& \leq \frac{\int_{\mathbb{R}^{n}}|\nabla u|^{p} d x-\left.\int_{\mathbb{R}^{n}}\left|\nabla u^{\star}\right|\right|^{p} d x}{\left.\int_{\left\{u^{\star}>u^{*}(1)\right\}}\left|\nabla u^{\star}\right|\right|^{p} d x} \\
& \leq \frac{\delta}{\left.C_{2}(p, n)^{p / \eta}\|u\|_{L^{1}\left(\mathbb{R}^{n}\right)}^{\frac{(1-\eta) p}{\eta}} \int_{\left\{u^{\star}>u^{*}(1)\right\}}\left|\nabla u^{\star}\right|\right|^{p} d x} \\
& =\frac{C_{18} \delta}{\left(\left.\int_{\mathbb{R}^{n}}\left|\nabla u^{\star}\right|\right|^{p} d x\right)^{\frac{1-\eta}{\eta}} \int_{\left\{u^{\star}>u^{*}(1)\right\}}\left|\nabla u^{\star}\right|^{p} d x}
\end{aligned}
$$

for some constant $C_{18}$ depending only on $p$ and $n$. Here, the first inequality follows from (2.5) applied to $u_{2}$, the second inequality from (3.3), and the last equality from (3.1). Finally, by (3.9) and (3.13),

$$
\int_{\mathbb{R}^{n}}\left|u_{2}\right| d x=\int_{1}^{\infty} u^{*}(s) d s+u^{*}(1) \leq \delta+C_{14} \delta^{1-\eta / p^{\prime}}\left((1+\delta) / C_{2}(p, n)\right)^{\eta / p^{\prime}}
$$

Now, let us apply (2.10) with $u$ replaced by $u_{1}$, with $\sigma=\frac{1}{\theta} \delta^{\frac{1-\gamma}{p}}$, and with $r_{1}, r_{2}, r_{3}$ so small that $1-\frac{r_{3} n^{\prime}}{p^{\prime}}>0, \frac{1}{p}-\frac{r_{1} r_{3}}{\eta}>0$ and $\frac{1}{p}-\frac{r_{2} r_{3}}{\eta}>0$. Furthermore, we chose $\gamma$ satisfying $r_{2}-\frac{1-\gamma}{p}>0$. Thus, from (3.16)-(3.18) and from the estimate $\left|\left\{\left|u_{1}\right|>0\right\}\right| \leq 1$, we infer that a positive constant $C_{19}$, depending only on $p$ and $n$, 
exists such that

$$
\begin{aligned}
& \min _{ \pm} \inf _{x_{0}}\left\|u(\cdot) \pm u^{\star}\left(\cdot-x_{0}\right)\right\|_{L^{1}\left(\mathbb{R}^{n}\right)} \\
& \leq \min _{ \pm} \inf _{x_{0}}\left\|u_{1}(\cdot) \pm u_{1}^{\star}\left(\cdot-x_{0}\right)\right\|_{L^{1}\left(\mathbb{R}^{n}\right)}+2 \int_{\mathbb{R}^{n}}\left|u_{2}\right| d x \\
& \leq C_{19}\left[\delta^{\gamma r_{3}}\left(\int_{\left\{u^{\star}>u^{*}(1)\right\}}\left|\nabla u^{\star}\right|^{p} d x\right)^{\frac{1}{p}}+\delta^{\frac{(1-\gamma) r_{3} n^{\prime}}{p^{\prime}}}\left(\int_{\left\{u^{\star}>u^{*}(1)\right\}}\left|\nabla u^{\star}\right|^{p} d x\right)^{\frac{1}{p}-\frac{r_{3} n^{\prime}}{p p^{\prime}}}\right. \\
& \quad+\delta^{\frac{(1-\gamma) r_{3}}{p^{\prime}}}\left(\int_{\left\{u^{\star}>u^{*}(1)\right\}}\left|\nabla u^{\star}\right|^{p} d x\right)^{\frac{1}{p}-\frac{r_{3}}{p p^{\prime}}} \\
& \quad+\delta^{r_{1} r_{3}}\left(\int_{\mathbb{R}^{n}}\left|\nabla u^{\star}\right|^{p} d x\right)^{\left(1-\frac{1}{\eta}\right) r_{1} r_{3}}\left(\int_{\left\{u^{\star}>u^{*}(1)\right\}}\left|\nabla u^{\star}\right|^{p} d x\right)^{\frac{1}{p}-r_{1} r_{3}} \\
& \left.\quad+\delta^{r_{3}\left(r_{2}-\frac{1-\gamma}{p}\right)}\left(\int_{\mathbb{R}^{n}}\left|\nabla u^{\star}\right|^{p} d x\right)^{\left(1-\frac{1}{\eta}\right) r_{2} r_{3}}\left(\int_{\left\{u^{\star}>u^{*}(1)\right\}}\left|\nabla u^{\star}\right|^{p} d x\right)^{\frac{r_{3}}{p^{2}}+\frac{1}{p}-r_{2} r_{3}}\right] \\
& \quad+2 \delta+2 C_{14} \delta^{1-\eta / p^{\prime}}\left((1+\delta) / C_{2}(p, n)\right)^{\eta / p^{\prime}} .
\end{aligned}
$$

Note that, owing to our choice of $r_{1}, r_{2}, r_{3}$ and $\gamma$, all the exponents of $\delta$ and of $\int_{\left\{u^{\star}>u^{*}(1)\right\}}\left|\nabla u^{\star}\right|^{p} d x$ in (3.19) are positive. Furthermore, $\left(1-\frac{1}{\eta}\right) r_{1} r_{3}+\frac{1}{p}-r_{1} r_{3}>0$ and $\left(1-\frac{1}{\eta}\right) r_{2} r_{3}+\frac{r_{3}}{p^{2}}+\frac{1}{p}-r_{2} r_{3}>0$. Consequently, since, by (3.1) and (3.2),

$$
\int_{\left\{u^{\star}>u^{*}(1)\right\}}\left|\nabla u^{\star}\right|^{p} d x \leq \int_{\mathbb{R}^{n}}\left|\nabla u^{\star}\right|^{p} d x \leq \int_{\mathbb{R}^{n}}|\nabla u|^{p} d x \leq C_{20}(1+\delta)^{\eta}
$$

for some positive constant $C_{20}$, depending only on $p$ and $n$, inequality (3.19) ensures that

$$
\min _{ \pm} \inf _{x_{0}}\left\|u(\cdot) \pm u^{\star}\left(\cdot-x_{0}\right)\right\|_{L^{1}\left(\mathbb{R}^{n}\right)} \leq C_{21} \sum_{i=1}^{7} \delta^{v_{i}}(1+\delta)^{\rho_{i}}
$$

for some positive constants $C_{21}$ and $v_{i}, \rho_{i}, i=1, \ldots, 7$, depending only on $p$ and $n$. From (3.14), (3.21) and (1.5), one can conclude that

$$
\inf _{a, b, x_{0}}\left\|u-w_{a, b, x_{0}}\right\|_{L^{\infty}\left(\mathbb{R}^{n}\right)} \leq C_{22} \delta^{v}
$$

for some positive constants $C_{22}$ and $v$, depending only on $p$ and $n$, and for every $u$ satisfying the additional constraints (2.12), (3.1) and $\delta \leq 1$. A similar argument as at the end of the proof of Theorem 1.1 eventually leads to (1.10) for every $u$ as in the statement.

\section{REFERENCES}

[Ad] R.A.Adams, "Sobolev spaces", Academic Press, Orlando, 1975. MR0450957 (56:9247)

[A] T.Aubin, Problèmes isopérimetriques et espaces de Sobolev, J. Diff. Geom 11 (1976), 573-598. MR0448404 (56:6711)

[BFT] G.Barbatis, S.Filippas \& A.Tertikas, A unified approach to improved $L^{p}$ Hardy inequalities with best constants, Trans. Amer. Math. Soc. 356 (2004), 2169-2196. MR2048514 (2005a:26016)

[BWW] T.Bartsch, T.Weth \& M.Willem, A Sobolev inequality with remainder term and critical equations on domains with topology for the polyharmonic operator, Calc. Var. Part. Diff. Equat. 18 (2003), 57-75. MR2018667(2004h:35059) 
[BE] G.Bianchi \& H.Egnell, A note on the Sobolev inequality, J. Funct. Anal. 100 (1991), 18-24. MR.1124290 (92i:46033)

[BL] H.Brezis \& E.Lieb, Sobolev inequalities with remainder terms, J. Funct. Anal. 62 (1985), 73-86. MR790771 (86i:46033)

[BN] H.Brezis \& L.Nirenberg, Positive solutions of nonlinear elliptic equations involving critical Sobolev exponents, Comm. Pure Appl. Math. 36 (1983), 437-477. MR709644 (84h:35059)

[BZ] J.E.Brothers \& W.P.Ziemer, Minimal rearrangements of Sobolev functions, J. Reine Angew. Math 384 (1988), 153-179. MR929981 (89g:26013)

[C] A. Cianchi, A quantitative Sobolev inequality in BV, J. Funct. Anal. 237 (2006), 466481. MR2230346 (2007b:46053)

[CEFT] A.Cianchi, L.Esposito, N.Fusco \& C.Trombetti, A quantitative Pólya-Szegö principle, J. Reine Angew. Math. 614 (2008).

[CF] A.Cianchi \& N.Fusco, Dirichlet integrals and Steiner asymmetry, Bull. Sci. Math. 130 (2006), 675-696. MR 2276198

[CFMP] A.Cianchi, N.Fusco, F.Maggi \& A.Pratelli, The sharp Sobolev inequality in quantitative form, preprint.

[DHA] A.Detella, T.Horiuchi \& H.Ando, Missing terms in Hardy-Sobolev inequalities and its applications, Far East J. Math. Sci. 14 (2004), 333-359. MR2108051 (2005h:26027)

[FF] H.Federer \& W.Fleming, Normal and integral currents, Annals of Math. 72 (1960), 458520. MR.0123260 (23:A588)

[FV] A.Ferone \& R.Volpicelli, Minimal rearrangements of Sobolev functions: a new proof, Ann. Inst. H.Poincaré, Anal. Nonlinéaire 20 (2003), 333-339. MR1961519 (2004c:46051)

[FMT] S.Filippas, V.G.Maz'ya \& A.Tertikas, On a question of Brezis and Marcus, Calc. Var. Part. Diff. Equat. 25 (2006), 491-501. MR2214621 (2006m:26046)

[GGS] F.Gazzola, H.C.Grunau \& M.Squassina, Existence and non existence results for critical growth biharmonic elliptic equations, Calc. Var. Part. Diff. Equat. 18 (2003), 117-143. MR 2010961 (2004j:35083)

$[\mathrm{H}] \quad$ K.Hilden, Symmetrization of functions in Sobolev spaces and the isoperimetric inequality, Manus. Math. 18 (1976), 215-235. MR0409773 (53:13525)

[K] B.Kawohl, Rearrangements and convexity of level sets in PDE, Lecture Notes in Math. 1150, Springer-Verlag, Berlin, 1985. MR810619 (87a:35001)

[M1] V.G.Maz'ya, Classes of regions and imbedding theorems for function spaces, Dokl. Akad. Nauk. SSSR 133 (1960), 527-530 (Russian); English translation: Soviet Math. Dokl. 1 (1960), 882-885. MR0126152 (23:A3448)

[M2] V.G.Maz'ya, "Sobolev spaces", Springer-Verlag, Berlin, 1985. MR817985 (87g:46056)

[S] E. Sperner, Symmetrisierung für Funktionen mehrerer reeller Variablen, Manus. Math. 11 (1974), 159-170. MR0328000 (48:6342)

[T1] G. Talenti, Best constant in Sobolev inequality, Ann. Mat. Pura Appl. 110 (1976), 353372. MR0463908 (57:3846)

[T2] G.Talenti, Inequalities in rearrangement invariant function spaces, in Nonlinear analysis, function spaces and applications, Vol. 5, M.Krbec, A.Kufner, B.Opic and J.Rákosnik Eds., Prometheus Publishing House, Prague, 1994. MR.1322306 (95i:00033)

[Z] W.P.Ziemer, "Weakly differentiable functions", Springer-Verlag, New York, 1989. MR 1014685 (91e:46046)

Dipartimento di Matematica e Applicazioni per l'Architettura, Università di Firenze, Piazza Ghiberti 27, 50122 Firenze, Italy

E-mail address: cianchi@unifi.it 